\title{
Comparison between Mother, ActiGraph wGT3X-BT, and a hand tally for measuring steps at various walking speeds under controlled conditions
}

\author{
Henrik Riel Corresp.. 1, 2, Pernille Møller Kalstrup ${ }^{2}$, Niels Kragh Madsen ${ }^{2}$, Elena Selmar Pedersen ${ }^{2}$, Camilla \\ Rams Rathleff ${ }^{2}$, Louise Bilenberg Pape-Haugaard ${ }^{2}$, Morten Villumsen ${ }^{2,3}$ \\ ${ }^{1}$ Research Unit for General Practice in Aalborg and Department of Clinical Medicine, Aalborg University, Aalborg, Denmark \\ 2 Department of Health Science and Technology, Aalborg University, Aalborg, Denmark \\ 3 Department of Physiotherapy, University College of Northern Denmark, Aalborg, Denmark \\ Corresponding Author: Henrik Riel \\ Email address: hriel@dcm.aau.dk
}

Introduction: Walking is endorsed as health enhancing and is the most common type of physical activity among older adults. Accelerometers are superior to self-reports when measuring steps however, if they are to be used by clinicians the validity is of great importance. The aim of this study was to investigate the criterion validity of Mother and ActiGraph wGT3X-BT in measuring steps by comparing the devices to a hand tally under controlled conditions in healthy participants. Methods: Thirty healthy participants were fitted with a belt containing the sensor of Mother (Motion Cookie) and ActiGraph. Participants walked on a treadmill for two minutes at each of the following speeds; 3.2 , 4.8 , and $6.4 \mathrm{~km} / \mathrm{h}$. The treadmill walking was video recorded and actual steps were subsequently determined by using a hand tally. Wilcoxon's signed ranks test was used to determine whether Mother and ActiGraph measured an identical number of steps compared to the hand tally. Intraclass correlation coefficients were calculated to determine the relationship and Root Mean Square error was calculated to investigate the average error between the devices and the hand tally. Percent differences (PD) were calculated for between-instrument agreement (Mother vs. the hand tally and ActiGraph vs. the hand tally) and PDs below 3\% were interpreted as acceptable and clinically irrelevant. Results: Mother and ActiGraph under-counted steps significantly compared to the hand tally at all walking speeds $(p<0.001)$. Mother had a median of total differences of 9.5 steps $(\mathrm{IQR}=10)$ and ActiGraph 59 steps $(\mathrm{IQR}=77)$. Mother had smaller PDs at all speeds especially at $3.2 \mathrm{~km} / \mathrm{h}(2.5 \%$ compared to $26.7 \%)$. Mother showed excellent ICC values at all speeds $\geq 0.88$ (0.51-0.96) whilst ActiGraph had poor and fair to good ICC values ranging from $0.03(-0.09-0.21)$ at a speed of $3.2 \mathrm{~km} / \mathrm{h}$ to $0.64(0.16-0.84)$ at a speed of $6.4 \mathrm{~km} / \mathrm{h}$. Conclusion: Mother provides valid measures of steps at walking speeds of 3.2, 4.8, and $6.4 \mathrm{~km} / \mathrm{h}$ with clinically irrelevant deviations compared to a hand tally while ActiGraph only 
provides valid measurements at $6.4 \mathrm{~km} / \mathrm{h}$ based on the $3 \%$ criterion. These results have significant potential for valid objective measurements of low walking speeds. However, further research should investigate the validity of Mother in patients at even slower walking speeds and in free-living conditions. 
1 Title: "Comparison between Mother, ActiGraph wGT3X-BT, and a hand tally

2 for measuring steps at various walking speeds under controlled conditions"

3 Authors: Henrik Riel ${ }^{1,2}$, Pernille Møller Kalstrup ${ }^{1}$, Niels Kragh Madsen ${ }^{1}$, Elena Selmar

4 Pedersen $^{1}$, Camilla Rams Rathleff ${ }^{1}$, Louise Bilenberg Pape-Haugaard ${ }^{1}$, Morten Villumsen ${ }^{1,3}$

5

$6{ }^{1}$ Department of Health Science and Technology, Faculty of Medicine, Aalborg University,

7 Fredrik Bajers Vej 5, 9220 Aalborg East, Denmark.

$8 \quad{ }^{2}$ Research Unit for General Practice in Aalborg and Department of Clinical Medicine, Aalborg

9 University, Fyrkildevej 7, 9220 Aalborg East, Denmark.

$10{ }^{3}$ Department of Physiotherapy, University College of Northern Denmark, Selma Lagerløfs Vej 2,

119220 Aalborg East, Denmark

12 Correspondence: Henrik Riel, hriel@dcm.aau.dk, Research Unit for General Practice in

13 Aalborg, Fyrkildevej 7, 9220 Aalborg, Denmark 
14 Abstract

15

16

17

Introduction: Walking is endorsed as health enhancing and is the most common type of physical activity among older adults. Accelerometers are superior to self-reports when measuring steps however, if they are to be used by clinicians the validity is of great importance. The aim of this study was to investigate the criterion validity of Mother and ActiGraph wGT3X-BT in measuring steps by comparing the devices to a hand tally under controlled conditions in healthy participants.

Methods: Thirty healthy participants were fitted with a belt containing the sensor of Mother (Motion Cookie) and ActiGraph. Participants walked on a treadmill for two minutes at each of the following speeds; $3.2,4.8$, and $6.4 \mathrm{~km} / \mathrm{h}$. The treadmill walking was video recorded and actual steps were subsequently determined by using a hand tally. Wilcoxon's signed ranks test was used to determine whether Mother and ActiGraph measured an identical number of steps compared to the hand tally. Intraclass correlation coefficients were calculated to determine the relationship and Root Mean Square error was calculated to investigate the average error between the devices and the hand tally. Percent differences (PD) were calculated for between-instrument agreement (Mother vs. the hand tally and ActiGraph vs. the hand tally) and PDs below 3\% were interpreted as acceptable and clinically irrelevant.

Results: Mother and ActiGraph under-counted steps significantly compared to the hand tally at all walking speeds $(\mathrm{p}<0.001)$. Mother had a median of total differences of 9.5 steps $(\mathrm{IQR}=10)$ and ActiGraph 59 steps (IQR=77). Mother had smaller PDs at all speeds especially at $3.2 \mathrm{~km} / \mathrm{h}$ (2.5\% compared to $26.7 \%)$. Mother showed excellent ICC values at all speeds $\geq 0.88(0.51-0.96)$ 
35 whilst ActiGraph had poor and fair to good ICC values ranging from $0.03(-0.09-0.21)$ at a speed

36 of $3.2 \mathrm{~km} / \mathrm{h}$ to $0.64(0.16-0.84)$ at a speed of $6.4 \mathrm{~km} / \mathrm{h}$.

37 Conclusion: Mother provides valid measures of steps at walking speeds of $3.2,4.8$, and $6.4 \mathrm{~km} / \mathrm{h}$

38 with clinically irrelevant deviations compared to a hand tally while ActiGraph only provides

39 valid measurements at $6.4 \mathrm{~km} / \mathrm{h}$ based on the $3 \%$ criterion. These results have significant

40 potential for valid objective measurements of low walking speeds. However, further research

41 should investigate the validity of Mother in patients at even slower walking speeds and in free-

42 living conditions. 


\section{Introduction}

44 Physical activity (PA) is endorsed as health enhancing (Steeves et al. 2015) and is known to

45 prevent and reduce both musculoskeletal disorders and mortality (Holtermann et al. 2012;

46 Holtermann et al. 2013; Haskell et al. 2007; Blair \& Morris 2009). Additionally, in older adults,

47 PA is especially important in maintaining self-dependence, preventing disease and improving the

48 quality of life (NHS Choices 2015). In contrast, the lack of PA is related to muscular alterations

49 such as atrophy and decreased muscle strength (Convertino et al. 1997; Appell 1990), thus

50 possibly contributing to loss of self-dependence, especially in older adults. During

51 hospitalization geriatric patients have shown low levels of PA (Villumsen et al. 2015) and only

$5217.8 \%$ of patients regain their pre-hospitalization level of mobility function 12 months after

53 admission (Visser et al. 2000). This emphasizes the need for PA awareness.

54 Walking is the most common type of leisure-time PA among adults and the prevalence of

55 walking for PA increases with age up until 65-74 years (Rafferty et al. 2002). In order to

56 measure PA, accelerometers are superior and recommended with respect to validity and

57 applicability (Müller et al. 2010) compared to self-reports, as patients often over- or

58 underestimate their actual level of PA (Sallis \& Saelens 2000; Farni et al. 2014; Barriera et al.

59 2013). Even though studies have found the validity and specificity to be high when measuring

60 different types of PA (i.e., moderate and high pace walking), accelerometers are considered

61 inadequate when measuring steps at low walking speeds (Crouter et al. 2013; Turner et al. 2012;

62 Steeves et al. 2011; Webber et al. 2014; Dijkstra et al. 2008; Barriera et al. 2013). If

63 accelerometers are to be successfully used by patients and/or healthcare personnel it may be of

64 importance that the accelerometers are valid, versatile, user-friendly, and inexpensive. 
65 One of the most commonly used accelerometers for monitoring PA is ActiGraph (ActiGraph,

66 Fort Walton, FL, USA) (Crouter et al. 2013; Barriera et al. 2013; Ekblom et al. 2012; Herman

67 Hansen et al. 2014). However, ActiGraph is developed with the intention to be used by

68 physicians and in research (ActiGraph 2015) whilst a new accelerometer, Mother (Sen.se, Paris,

69 France), is developed with the intention to be used by the private consumer (Sen.se 2015a).

70 The aim of this study was to investigate the criterion validity of Mother (Sen.se, Paris, France)

71 and ActiGraph wGT3X-BT (ActiGraph, Fort Walton, FL, USA) in measuring steps by

72 comparing the devices to a hand tally, which is considered gold standard, under controlled

73 conditions in healthy participants.

\section{Methods}

75 This study is a validity study that complies with the Guidelines for Reporting Reliability and

76 Agreement Studies (GRRAS) (Kottner et al. 2011).

\section{Ethics statement}

78 Ethical approval of the research protocol was not needed according to The North Denmark

79 Region Committee on Health Research Ethics. Written informed consent was signed by all

80 participants prior to the study. 


\section{Sample size and raters}

82 Sample size was determined to be 30 participants using large sample case (Hogg \& Tanis 1996).

83 Two raters performed the hand tallying and conducted the treadmill test whilst two different

84 raters performed the data treatment without being part of the data collection.

\section{Participants}

86 Thirty-one healthy students were recruited from the Aalborg University, Denmark (male n=15,

87 female $n=16$ ). Data was collected from March to April 2015.

88 The inclusion criteria were: i) age of 18 years or above, ii) no self-reported health problems

89 evaluated by the Physical Activity Readiness Questionnaire (PAR-Q), iii) ability to walk without

90 walking aids, iv) ability to walk continuously for 10 minutes on a treadmill, and v) ability to

91 read, understand and speak Danish and English. The fifth criterion was chosen to ensure that

92 participants understood the instructions and the PAR-Q, which was in English, as no Danish

93 translation was available.

94 The exclusion criteria were: i) pregnancy (self-reported), ii) BMI $\geq 30 \mathrm{~kg} / \mathrm{m}^{2}$, and iii)

95 neurological diseases (self-reported).

96 Thirty-one healthy participants were recruited for the study. One participant was excluded due to

97 a BMI $>30 \mathrm{~kg} / \mathrm{m}^{2}$. Participants did not report any impairments or morbidities potentially

98 interfering with the assessment. See table 1 for participant demographics.

99 Table 1: Participant demographics

100 Mother 
101 Mother (Sen.se, Paris, France) is a triaxial accelerometer released in March 2014 by Sen.se

102 (Sen.se 2014). The device consists of a hub (Mother), up to 24 sensors (Motion Cookies), and a 103 software application (the Senseboard) (Sen.se, Paris, France). User access is gained through the 104 Senseboard, which is a collection of different applications developed by Sen.se. The dimensions 105 of a Motion Cookie are 5.0*2.2*0.4 cm with a weight of 6 grams (Sen.se 2015b). The sample 106 rate is fixed at $25 \mathrm{~Hz}$. The accelerometer has a dynamic range of $\pm 2 \mathrm{G}$ and a precision of $12 \mathrm{bit}$ 107 (Alain Romanet, e-mail correspondence with Sen.se, March $9^{\text {th }}$ 2016). In this study the 108 application Walk (Sen.se, Paris, France) was selected for measuring steps. Data from the Motion 109 Cookie is uploaded to Mother every 5 minutes (Franck Biehler, e-mail correspondence with 110 Sen.se, March $23^{r d} 2015$ ) in fractions of varying durations.

\section{ActiGraph wGT3X-BT}

ActiGraph wGT3X-BT (ActiGraph LLC, Pensacola, FL, USA) is a triaxial accelerometer and one of the most commonly used devices for assessing PA (Crouter et al. 2013). The dimensions of the sensor are $4.6 * 3.3 * 1.5 \mathrm{~cm}$ with a weight of 19 grams. A sample rate of $100 \mathrm{~Hz}$ was chosen (range 30-100 Hz). The accelerometer has a dynamic range of $\pm 8 \mathrm{G}$ and a precision of 12 bit (ActiGraph 2015). Data is accessible by using the ActiLife Pro 6 software (ActiGraph LLC, Pensacola, FL, USA). After the recordings the sensor was connected to a computer through a mini-USB cable in order to upload the data. During initialization, information including subject name, gender, height, weight, race, limb and leg dominance is required, however random values were used as step counting is not affected by this information (ActiGraph 2015). 


\section{Accelerometer placement}

122 The participants wore Mother's Motion Cookie and ActiGraph simultaneously placed on an

123 elastic belt above the right anterior superior iliac spine (SIAS) $(0.5 \mathrm{~cm}$. medially and laterally

124 from the right SIAS, respectively) (Figure 1). Hip placement has previously been found as the

125 most precise single location placement of an accelerometer (Cleland et al. 2013). The placement

126 of devices was randomized using a random number generator (www.random.org)

127 (Randomization 0: Mother laterally, Randomization 1: ActiGraph laterally) to take possible

128 placement related differences in validity into account. 17 participants received Randomization 0

129 and 13 participants received Randomization 1.

Figure 1: Accelerometer placement

Note. ActiGraph is placed laterally to the right SIAS and the Motion Cookie is placed medially (Randomization 1)

\section{Hand tally}

133 A hand tally is considered gold standard when measuring steps (Dijkstra et al. 2008; Fortune et 134 al. 2014; Stemland et al. 2015). In this study, the application AGR Tally counter (ver. 1.0, Angel

135 Garcia Rubio) was used for hand tallying. Steps were measured by tapping the screen of an 136 iPhone 4s (Apple Inc., Infinite Loop Cupertino, California, USA).

\section{Procedures of the treadmill test}

138 The number of steps was obtained during a treadmill test where participants walked on a

139 treadmill for two minutes at each of the three walking speeds; $3.2,4.8$, and $6.4 \mathrm{~km} / \mathrm{h}$. The

140 inclination was set to $0^{\circ}$. The treadmill was preprogramed to standardize the procedure for an in-

141 and decrease of the walking speed. The two minutes included the time the treadmill in- and 142 decreased the walking speed. The speeds were chosen in accordance with walking speeds chosen 
143 in three previous studies that investigated the validity of measuring steps under controlled

144 conditions at various walking speeds (Steeves et al. 2011; De Cocker et al. 2012; Clemes et al.

145 2010). The test was conducted in the sports science laboratory at Aalborg University, Denmark.

146 To take the inability of Mother to synchronize more often than every 5 minutes into

147 consideration, the participants were asked to stand still for five minutes and 10 seconds before

148 and after each walking speed in order to identify the walking session in the application

149 programming interface (API).

150

151

152

153

154

155

156

157

158

159

160

161

162

163

\section{Data Treatment}

Data from Mother for each test were identified by examining the walking duration in the API. Even though the participants walked for exactly two minutes, which was confirmed by video recordings of the tests, walking sessions had durations ranging from 115-130 seconds. The output from the API showing the number of steps of the walking session was manually to identify equipment malfunctions such as missing steps. Accelerometer data from ActiGraph were downloaded using ActiLife 6 Pro software.

Video recordings of the treadmill test were used for hand tallying. The definition of a step was adopted by Dijkstra et al. and defined as "the first moment at which the heel of the foot for the initial step cleared the ground and the moment at which the foot of the closing step made completely contact with the floor" (Dijkstra et al. 2008). The hand tallying procedure was double validated as the two raters hand tallied independently. The two raters had $100 \%$ agreement. The two raters responsible for data treatment and the statistical analyses had not been involved in the treadmill test nor the hand tallying. 


\section{Statistical analysis}

165 All statistical analyses were performed using IBM SPSS (ver. 22, IBM Corporation, New York, 166 United States) with a significance level of $\mathrm{p}<0.05$.

167 Normal distribution was examined based on the differences between the number of steps

168 measured by Mother and the hand tally and ActiGraph and the hand tally using Q-Q plots and

169 Shapiro-Wilk test. Q-Q plots were assessed and as data did not appear to be normally distributed,

170 a Shapiro-Wilk test was performed and confirmed that data were non-normally distributed $171(\mathrm{p}<0.05)(\mathrm{DOI}: 10.6084 / \mathrm{m} 9$. figshare.3814272.v1).

172 Wilcoxon's signed ranks test was used to create a pairwise comparison to determine whether the 173 devices and the hand tally measured the same number of steps. Means and standard deviations 174 (SD) were computed for age, height, weight and BMI whilst medians and interquartile ranges 175 (IQR) were computed for steps and differences in steps measured by the devices. A two-way 176 random effects model (2.1), single measures, absolute agreement, and intraclass correlation coefficients (ICC) with 95\% confidence intervals were used to express interrater reliability

178 between the devices and the hand tally. ICC values $>0.75$ were interpreted excellent, $0.40-0.75$

179 were interpreted fair to good and $<0.40$ were interpreted poor (Fleiss 2011). To investigate the average error Root Mean Square error (RMSe) was calculated between each device and the hand tally. As data were non-normally distributed the nonparametric approach to presenting BlandAltman plots was adopted and the median, the $2.5^{\text {th }}$, and the $97.5^{\text {th }}$ percentiles were visualised in the plots (Gialamas et al. 2010).

Percent differences (PD) were calculated for between-instrument agreement (Mother vs. the hand tally and ActiGraph vs. the hand tally). The PD was calculated as $\frac{\text { Absolute difference }}{\text { hand tally steps }} \cdot 100$. Any negative values were converted to a positive to calculate the absolute difference. Clinical 
187 relevance of potential under- or over-counting of steps by the two accelerometers compared to

188 hand tally was determined using a 3\% criterion, which was based on previous studies (Johnson et

189 al. 2015; Holbrook et al. 2009; Colley et al. 2013; Liu et al. 2015). PD $\leq 3 \%$ were considered

190 clinically irrelevant.

191 Results

192

\section{Mother vs. the hand tally}

193

194

195

196

197

198

199

200

201

202

203

204

205

206

207

208

209

The median of differences in steps between Mother and the hand tally at the different walking speeds were 2.5 steps $(I Q R=5)$ at $3.2 \mathrm{~km} / \mathrm{h}, 2$ steps $(\mathrm{IQR}=2)$ at $4.8 \mathrm{~km} / \mathrm{h}$ and 3.5 steps $(\mathrm{IQR}=6)$ at $6.4 \mathrm{~km} / \mathrm{h}$ (Table 2). These are depicted in the Bland-Altman plot (Figure 2). The median of total differences was 9.5 steps $(\mathrm{IQR}=10)$.

Table 2: The median of the number of steps measured by each device and the median of differences between the hand tally vs. Mother and the hand tally vs. ActiGraph

Wilcoxon's signed ranks test showed a significant difference in the number of steps measured by the hand tally vs. Mother at all walking speeds $(\mathrm{p}<0.001)$ (Table 3).

Table 3: Results from Wilcoxon's signed ranks test with significance levels of each comparison, intraclass correlation coefficient, Root Mean Square error, and percent difference. *Significant difference.

The ICCs for Mother and the hand tally were all excellent ranging from $0.88(0.51-0.96)$ at a speed of $3.2 \mathrm{~km} / \mathrm{h}$ to $0.96(0.72-0.99)$ at a speed of $4.8 \mathrm{~km} / \mathrm{h}$ (Table 3$)$. The RMSe ranged from 2.86 at $4.8 \mathrm{~km} / \mathrm{h}$ to 5.50 at $3.2 \mathrm{~km} / \mathrm{h}$ (Table 3 ). Mother had PDs $\leq 2.5 \%$ of the steps measured by the hand tally at all speeds (Table 3).

Figure 2: Bland-Altman style plot of differences between the actual number of steps and steps measured by Mother. The solid line depicts the median of differences and the dotted lines depict the $2.5^{\text {th }}$ and $97.5^{\text {th }}$ percentiles of each walking speed. The colour of the data point refers to the walking speed. 


\section{ActiGraph vs. the hand tally}

211 The median of differences in steps between ActiGraph and the hand tally at the different walking

212 speeds were 49.5 steps $(\mathrm{IQR}=69)$ at $3.2 \mathrm{~km} / \mathrm{h}, 4$ steps $(\mathrm{IQR}=5)$ at $4.8 \mathrm{~km} / \mathrm{h}$ and 4 steps $(\mathrm{IQR}=5)$

213 at $6.4 \mathrm{~km} / \mathrm{h}$ (Table 2). These are depicted in the Bland-Altman plot (Figure 3). The median of

214 total differences was 59 steps $(\mathrm{IQR}=77)$.

215 Wilcoxon's signed ranks test showed a significant difference in the number of steps measured by

216 the hand tally vs. ActiGraph at all walking speeds $(\mathrm{p}<0.001)$ (Table 3$)$.

217 The ICCs for ActiGraph and the hand tally were poor and fair to good ranging from 0.03 (-0.09-

$2180.21)$ at a speed of $3.2 \mathrm{~km} / \mathrm{h}$ to $0.64(0.16-0.84)$ at a speed of $6.4 \mathrm{~km} / \mathrm{h}$ (Table 3$)$. The RMSe

219 ranged from 8.80 at $6.4 \mathrm{~km} / \mathrm{h}$ to 36.52 at $3.2 \mathrm{~km} / \mathrm{h}$ (Table 3$)$.

220 ActiGraph had PDs $\leq 26.7 \%$ of the steps measured by the hand tally (Table 3 ). ActiGraph was

221 under-counting based on the $3 \%$ criterion at both 3.2 and $4.8 \mathrm{~km} / \mathrm{h}$.

222 Figure 3: Bland-Altman style plot of differences between the actual number of steps and steps measured by

223 ActiGraph. The solid line depicts the median of differences and the dotted lines depict the $2.5^{\text {th }}$ and $97.5^{\text {th }}$ percentiles

224 of each walking speed. The colour of the data point refers to the walking speed.

\section{Discussion}

226

227

228

229

230

This study aimed at investigating the criterion validity of Mother and ActiGraph in measuring steps by comparing the devices to a hand tally under controlled conditions in healthy participants. The results revealed that both Mother and ActiGraph under-counted steps significantly compared to the hand tally at all walking speeds and Mother had smaller PDs at all walking speeds compared to ActiGraph. 
231 Both Mother and ActiGraph under-counted steps significantly at all walking speeds compared to

232 the hand tally, which is considered gold standard of measuring steps. Hence, it is apparent that

233 none of these accelerometers have the same level of precision as the hand tally. However,

234 significant results are not always clinically relevant as it would be unrealistic to use a hand tally

235 under free-living conditions. Therefore, a 3\% clinically irrelevant deviation from the steps

236 measured by the hand tally was adopted inspired by previous studies of validity (Johnson et al.

237 2015; Holbrook et al. 2009; Colley et al. 2013; Liu et al. 2015). The PDs of $\leq 2.5 \%$ measured by

238 Mother were interpreted as being clinically irrelevant as it was less than 3\%, however ActiGraph

239 had clinically relevant deviations at both 3.2 and $4.8 \mathrm{~km} / \mathrm{h}$. Especially at $3.2 \mathrm{~km} / \mathrm{h}$ with a PD of

$24026.7 \%$ ActiGraph may not be adequately precise to measure steps in patients with a low walking

241 speed. These results are in accordance with previous findings of the validity of ActiGraph at

242 measuring steps at low walking speeds, which found that ActiGraph measured only $77.5 \%$ of the

243 actual steps at the speed of $3.2 \mathrm{~km} / \mathrm{h}$ (Connolly et al. 2011). In a practical perspective, ActiGraph

244 would under-count 4725 steps in a week if a patient has a daily average of 3000 steps which

245 would make it difficult for the clinician to determine whether or not the patient was following the

246 advised physical activity. Accelerometers in general are known for being inadequate when

247 measuring steps at the low speeds that some of the patients may walk at (Barriera et al. 2013;

248 Crouter et al. 2013; Turner et al. 2012; Steeves et al. 2011; Webber et al. 2014; Dijkstra et al.

249 2008), but even though Mother is an accelerometer developed with the intention to be used by

250 private consumers, it showed a superior accuracy compared to the accelerometer most commonly

251 used for measuring PA in research (Crouter et al. 2013).

252 The excellent ICC between Mother and the hand tally would make a prediction of a margin of

253 error at a given number of steps feasible. This means that with any given number of steps, the 
254 amount of miscounted steps can be estimated, thus making measurements with Mother more

255 valid even at a large step counts.

\section{Limitations}

257 The participants of this study were a group of younger, healthy subjects who performed steps

258 that were similar to the definition of a step by Dijkstra et al. (Dijkstra et al. 2008). However,

259 patients may walk asymmetric or without a swing phase which might provide different results.

260 Healthy participants were chosen, as the purpose of this study was to test the validity under

261 controlled conditions. Therefore, the results cannot be directly applied to any given patient

262 group.

263 The walking sessions in the API of Mother had varying durations ranging from 115-130 seconds.

264 The reason for this variation is unknown however, it implies that the participants have either

265 stopped walking prior to the end of the two minutes or they have continued moving even though

266 the treadmill had stopped. This was, however, not indicated by the video recordings. Another

267 explanation for the 115 second walking sessions could be that Mother stopped measuring as the

268 participant slowed down before coming to a halt. As Mother measured durations both shorter and

269 longer than the 120 seconds the walking session lasted, the inconsistencies in duration may have

270 evened out.

\section{Future work}

272 This study investigated step measuring at a walking speed of $3.2 \mathrm{~km} / \mathrm{h}$ as the lowest speed, but some patients may walk at an even slower pace, thus investigating the validity at lower walking

274 speeds is highly relevant to determine the minimum speed at which Mother still provides

275 measures of steps that have clinically irrelevant deviations from the actual number of steps. 
276 Future studies should also include testing in a semi-controlled environment and in free-living

277 conditions and should also include participants with a larger BMI than included in this study as

278 the waist circumference can influence precision due to tilting (Crouter et al. 2005).

\section{Conclusion}

280 Mother provides valid measures of steps at walking speeds of 3.2, 4.8, and $6.4 \mathrm{~km} / \mathrm{h}$ with

281 clinically irrelevant deviations compared to a hand tally while ActiGraph only provides valid

282 measurements at $6.4 \mathrm{~km} / \mathrm{h}$ based on the $3 \%$ criterion. These results have significant potential for

283 valid objective measurements of low walking speeds. 
284 References

285

286

287

288

289

290

291

292

ActiGraph, 2015. http://www.actigraphcorp.com/solutions-and-products/activitymonitors/actigraph-wgt3x-bt/. Available at: http://www.actigraphcorp.com/solutions-andproducts/activity-monitors/actigraph-wgt3x-bt/ [Accessed September 9, 2016].

Appell, H.J., 1990. Muscular atrophy following immobilisation. A review. Sports medicine (Auckland, N.Z.), 10(1), pp.42-58.

Barriera, T. V, Tudor-Locke, C., Champagne, C. M., Broyles, S. T., Johnson, W. D. and Katzmarzyk, P. T., 2013. Comparison of GT3X accelerometer and YAMAX pedometer steps/day in a free-living sample of overweight and obese adults. Journal of physical activity \& health, 10(2), pp.263-70.

Blair, S. N. and Morris, J. N., 2009. Healthy Hearts—and the Universal Benefits of Being Physically Active: Physical Activity and Health. Annals of Epidemiology, 19(4), pp.253256.

Cleland, I., Kikhia, B., Nugent, C., Boytsov, A., Hallberg, J., Synnes, K., McClean, S. and Finlay, D., 2013. Optimal placement of accelerometers for the detection of everyday activities. Sensors (Basel, Switzerland), 13(7), pp.9183-200.

Clemes, S. A., O’Connell, S., Rogan, L. M. and Griffiths, P. L., 2010. Evaluation of a commercially available pedometer used to promote physical activity as part of a national programme. British journal of sports medicine, 44(16), pp.1178-83.

De Cocker, K. A., De Meyer, J., De Bourdeaudhuij, I. M. and Cardon, G. M., 2012. Nontraditional wearing positions of pedometers: validity and reliability of the Omron HJ-203- 

in sport / Sports Medicine Australia, 15(5), pp.418-24.

307 Colley, R. C., Barnes, J. D., Leblanc, A. G., Borghese, M., Boyer, C. and Tremblay, M. S., 2013.

308 Validity of the SC-StepMX pedometer during treadmill walking and running. Applied

309 physiology, nutrition, and metabolism = Physiologie appliquée, nutrition et métabolisme,

310 38(5), pp.520-4.

311

312

313

314

315

316

317

318

319

320

321

322

323

324

325

Connolly, C. P., Coe, D. P., Kendrick, J. M., Bassett, D. R. and Thompson, D. L., 2011. Accuracy of physical activity monitors in pregnant women. Medicine and science in sports and exercise, 43(6), pp.1100-5.

Convertino, V.A., Bloomfield, S.A. \& Greenleaf, J.E., 1997. An overview of the issues: physiological effects of bed rest and restricted physical activity. Medicine and science in sports and exercise, 29(2), pp.187-190.

Crouter, S.E., Schneider, P.L. \& Bassett, D.R., 2005. Spring-levered versus piezo-electric pedometer accuracy in overweight and obese adults. Medicine and science in sports and exercise, 37(10), pp.1673-9.

Crouter, S. E., DellaValle, D. M., Haas, J. D., Frongillo, E. A. and Bassett, D. R., 2013. Validity of ActiGraph 2-regression model, Matthews cut-points, and NHANES cut-points for assessing free-living physical activity. Journal of physical activity \& health, 10(4), pp.504514.

Dijkstra, B., Zijlstra, W., Scherder, E. and Kamsma, Y., 2008. Detection of walking periods and number of steps in older adults and patients with Parkinson's disease: accuracy of a 

pedometer and an accelerometry-based method. Age and ageing, 37(4), pp.436-41.

327

Ekblom, O., Nyberg, G., Bak, E. E., Ekelund, U. and Marcus, C., 2012. Validity and comparability of a wrist-worn accelerometer in children. Journal of physical activity \& health, 9(3), pp.389-393.

Farni, K., Shoham, D. A., Cao, G., Luke, A. H., Layden, J., Cooper, R. S. and Dugas, L. R., 2014. Physical activity and pre-diabetes-an unacknowledged mid-life crisis: findings from NHANES 2003-2006. PeerJ, 2(Cvd), p.e499.

Fleiss, J.L., 2011. Design and analysis of clinical experiments Vol. 73., John Wiley \& Sons.

Fortune, E., Lugade, V., Morrow, M. and Kaufman, K., 2014. Validity of using tri-axial accelerometers to measure human movement - Part II: Step counts at a wide range of gait velocities. Medical engineering \& physics, 36(6), pp.659-69.

Gialamas, A., Laurence, C. O., Yelland, L. N., Tideman, P., Worley, P., Shephard, M. D., Tirimacco, R., Willson, K. J., Ryan, P., Gill, J., Thomas, D. W. and Beilby, J. J., 2010. Assessing agreement between point of care and laboratory results for lipid testing from a clinical perspective. Clinical Biochemistry, 43(4-5), pp.515-518.

Haskell, W. L., Lee, I.-M., Pate, R. R., Powell, K. E., Blair, S. N., Franklin, B. A., Macera, C. A., Heath, G. W., Thompson, P. D. and Bauman, A., 2007. Physical activity and public health: updated recommendation for adults from the American College of Sports Medicine and the American Heart Association. Medicine and science in sports and exercise, 39(8), pp.1423-34.

Herman Hansen, B., Bortnes, I., Hildebrand, M., Holme, I., Kolle, E. and Anderssen, S. A., 
2014. Validity of the ActiGraph GT1M during walking and cycling. Journal of sports sciences, 32(6), pp.510-516.

Hogg, R. V \& Tanis, E.A., 1996. Probability and Statistical Inference 5th edn., Upper Saddle River, NJ: Prentice Hall.

Holbrook, E.A., Barreira, T. V \& Kang, M., 2009. Validity and reliability of Omron pedometers for prescribed and self-paced walking. Medicine and science in sports and exercise, 41(3), pp.670-674.

Holtermann, A., Marott, J. L., Gyntelberg, F., Søgaard, K., Suadicani, P., Mortensen, O. S., Prescott, E. and Schnohr, P., 2013. Does the benefit on survival from leisure time physical activity depend on physical activity at work? A prospective cohort study. PloS one, 8(1), p.e54548.

Holtermann, A., Hansen, J. V., Burr, H., Sogaard, K. and Sjogaard, G., 2012. The health paradox of occupational and leisure-time physical activity. British Journal of Sports Medicine, 46(4), pp.291-295.

Johnson, M., Meltz, K., Hart, K., Schmudlach, M., Clarkson, L. and Borman, K., 2015. Validity of the Actical activity monitor for assessing steps and energy expenditure during walking. Journal of Sports Sciences, 33(8), pp.769-776.

Kottner, J., Audige, L., Brorson, S., Donner, A., Gajewski, B. J., Hrobjartsson, A., Roberts, C., Shoukri, M. and Streiner, D. L., 2011. Guidelines for Reporting Reliability and Agreement Studies (GRRAS) were proposed. International journal of nursing studies, 48(6), pp.661671. 
368 Liu, S., Brooks, D., Thomas, S., Eysenbach, G. and Nolan, R. P., 2015. Lifesource XL-18

369

370

371

372

373

374

375

376

377

378

379

380

381

382

383

384

385

386

387

388

pedometer for measuring steps under controlled and free-living conditions. Journal of sports sciences, 33(10), pp.1001-6.

Müller, C., Winter, C. \& Rosenbaum, D., 2010. Aktuelle objektive messverfahren zur erfassung körperlicher aktivität im vergleich zu subjektiven erhebungsmethoden. Deutsche Zeitschrift fur Sportmedizin, 61(1), pp.11-18.

NHS Choices, 2015. Physical activity guidelines for older adults. Available at: http://www.nhs.uk/Livewell/fitness/Pages/physical-activity-guidelines-for-older-adults.aspx [Accessed April 29, 2016].

Rafferty, A. P., Reeves, M. J., McGee, H. B. and Pivarnik, J. M., 2002. Physical activity patterns among walkers and compliance with public health recommendations. Medicine \& Science in Sports \& Exercise, 34(8), pp.1255-1261.

Sallis, J.F. \& Saelens, B.E., 2000. Assessment of physical activity by self-report: Status, limitations, and future directions. Research Quarterly for Exercise and Sport, 71(2), pp.114.

Sen.se, 2015a. https://sen.se/about/offer/. Available at: https://sen.se/about/offer/ [Accessed April 29, 2016].

Sen.se, 2015b. https://sen.se/store/mother/. , (13-5). Available at: https://sen.se/store/mother/ [Accessed April 29, 2016].

Sen.se, 2014. Introducing Mother,Reinvented - Sen.se Creates a Caring, Adaptable and Programmable Mom that Covers all the Areas of Your Life. , 1. 
389 Steeves, J. A., Tudor-Locke, C., Murphy, R. A., King, G. A., Fitzhugh, E. C. and Harris, T. B., 390 2015. Classification of occupational activity categories using accelerometry: NHANES 2003-2004. The international journal of behavioral nutrition and physical activity, 12(1),

Steeves, J. A., Tyo, B. M., Connolly, C. P., Gregory, D. A., Stark, N. A. and Bassett, D. R., 2011. Validity and reliability of the Omron HJ-303 tri-axial accelerometer-based pedometer. Journal of physical activity \& health, 8(7), pp.1014-1020.

Stemland, I., Ingebrigtsen, J., Christiansen, C. S., Jensen, B. R., Hanisch, C., Skotte, J. and Holtermann, A., 2015. Validity of the Acti4 method for detection of physical activity types in free-living settings: comparison with video analysis. Ergonomics, 58(6), pp.953-965.

Turner, L. J., Houchen, L., Williams, J. and Singh, S. J., 2012. Reliability of pedometers to measure step counts in patients with chronic respiratory disease. Journal of cardiopulmonary rehabilitation and prevention, 32(5), pp.284-291.

Villumsen, M., Jorgensen, M. G., Andreasen, J., Rathleff, M. S. and Mølgaard, C. M., 2015. Very Low Levels of Physical Activity in Older Patients During Hospitalization at an Acute Geriatric Ward: A Prospective Cohort Study. Journal of aging and physical activity, 23(4),

Visser, M., Harris, T. B., Fox, K. M., Hawkes, W., Hebel, J. R., Yahiro, J. Y., Michael, R., 407 pp.542-9. Zimmerman, S. I. and Magaziner, J., 2000. Change in muscle mass and muscle strength after a hip fracture: relationship to mobility recovery. The journals of gerontology.Series A, Biological sciences and medical sciences, 55(8), pp.M434-40. 
410 Webber, S. C., Magill, S. M., Schafer, J. L. and Wilson, K. C., 2014. GT3X+ accelerometer,

411 Yamax pedometer and SC-StepMX pedometer step count accuracy in community-dwelling

$412 \quad$ older adults. , 22(3), p.334.

413 


\section{Table 1 (on next page)}

\section{Participant demographics}


1

\begin{tabular}{ll}
\hline Gender (N, men/women) & $15 / 15$ \\
Age (years, mean (SD)) & $27.9( \pm 4.2)$ \\
Height (cm, mean (SD)) & $173.5( \pm 9.1)$ \\
Weight $(\mathrm{kg}$, mean $(\mathrm{SD}))$ & $71.6( \pm 11.3)$ \\
BMI $\left(\mathrm{kg} / \mathrm{m}^{2}\right.$, mean $\left.(\mathrm{SD})\right)$ & $23.6( \pm 2.2)$ \\
\hline
\end{tabular}

2 


\section{Table 2 (on next page)}

The median of the number of steps measured by each device and the median of differences between the hand tally vs. Mother and the hand tally vs. ActiGraph 


\begin{tabular}{|c|c|c|c|}
\hline & $\begin{array}{l}\text { Walking speed } \\
\qquad(\mathrm{km} / \mathrm{h})\end{array}$ & Median of steps & $\begin{array}{l}\text { Hand tally } \\
\text { Median of } \\
\text { differences in } \\
\text { steps }\end{array}$ \\
\hline \multirow[t]{4}{*}{ Hand tally } & 3.2 & $190(\mathrm{IQR}=13)$ & - \\
\hline & 4.8 & $229(\mathrm{IQR}=11)$ & - \\
\hline & 6.4 & $260(\mathrm{IQR}=14)$ & - \\
\hline & Total & $677(\mathrm{IQR}=35)$ & - \\
\hline \multirow[t]{4}{*}{ Mother } & 3.2 & $186(\mathrm{IQR}=22)$ & $2.5(\mathrm{IQR}=5)$ \\
\hline & 4.8 & $227(\mathrm{IQR}=12)$ & $2(\mathrm{IQR}=2)$ \\
\hline & 6.4 & $254(\mathrm{IQR}=17)$ & $3.5(\mathrm{IQR}=6)$ \\
\hline & Total & $663(\mathrm{IQR}=43)$ & $9.5(\mathrm{IQR}=10)$ \\
\hline \multirow[t]{4}{*}{ ActiGraph } & 3.2 & $134.5(\mathrm{IQR}=70)$ & $49.5(\mathrm{IQR}=69)$ \\
\hline & 4.8 & $222(\mathrm{IQR}=10)$ & $4(\mathrm{IQR}=5)$ \\
\hline & 6.4 & $254(\mathrm{IQR}=15)$ & $4(\mathrm{IQR}=5)$ \\
\hline & Total & $615(\mathrm{IQR}=73)$ & $59(\mathrm{IQR}=77)$ \\
\hline
\end{tabular}

1 


\section{Table 3(on next page)}

Results from Wilcoxon's signed ranks test with significance levels of each comparison, intraclass correlation coefficient, Root Mean Square error, and percent difference. *Significant difference. 


\begin{tabular}{|c|c|c|c|}
\hline & $\begin{array}{l}\text { Walking } \\
\text { speed } \\
(\mathrm{km} / \mathrm{h})\end{array}$ & Mother vs. Hand tally & ActiGraph vs. Hand tally \\
\hline \multirow[t]{4}{*}{ Wilcoxon's signed ranks test } & 3.2 & $\mathrm{p}<0.001^{*}$ & $\mathrm{p}<0.001^{*}$ \\
\hline & 4.8 & $\mathrm{p}<0.001 *$ & $\mathrm{p}<0.001^{*}$ \\
\hline & 6.4 & $\mathrm{p}<0.001^{*}$ & $\mathrm{p}<0.001^{*}$ \\
\hline & Total & $\mathrm{p}<0.001^{*}$ & $\mathrm{p}<0.001^{*}$ \\
\hline \multirow{4}{*}{$\begin{array}{l}\text { Intraclass correlation coefficient } \\
\qquad(\mathrm{ICC}(95 \% \mathrm{CI}))\end{array}$} & 3.2 & $0.88(0.51-0.96)$ & $0.03(-0.09-0.21)$ \\
\hline & 4.8 & $0.96(0.72-0.99)$ & $0.55(0.13-0.78)$ \\
\hline & 6.4 & $0.89(0.19-0.97)$ & $0.64(0.16-0.84)$ \\
\hline & Total & $0.93(0.18-0.98)$ & $0.22(-0.10-0.54)$ \\
\hline \multirow[t]{4}{*}{ RMSe } & 3.2 & 5.50 & 36.52 \\
\hline & 4.8 & 2.86 & 11.66 \\
\hline & 6.4 & 3.88 & 8.80 \\
\hline & Total & 8.33 & 48.18 \\
\hline \multirow[t]{4}{*}{$\mathrm{PD}(\%)$} & 3.2 & 2.5 & 26.7 \\
\hline & 4.8 & 1.3 & 3.7 \\
\hline & 6.4 & 1.9 & 2.8 \\
\hline & Total & 1.8 & 9.8 \\
\hline
\end{tabular}

1 


\section{Figure 1}

\section{Accelerometer placement}

Note. ActiGraph is placed laterally to the right SIAS and the Motion Cookie is placed medially (Randomization 1)

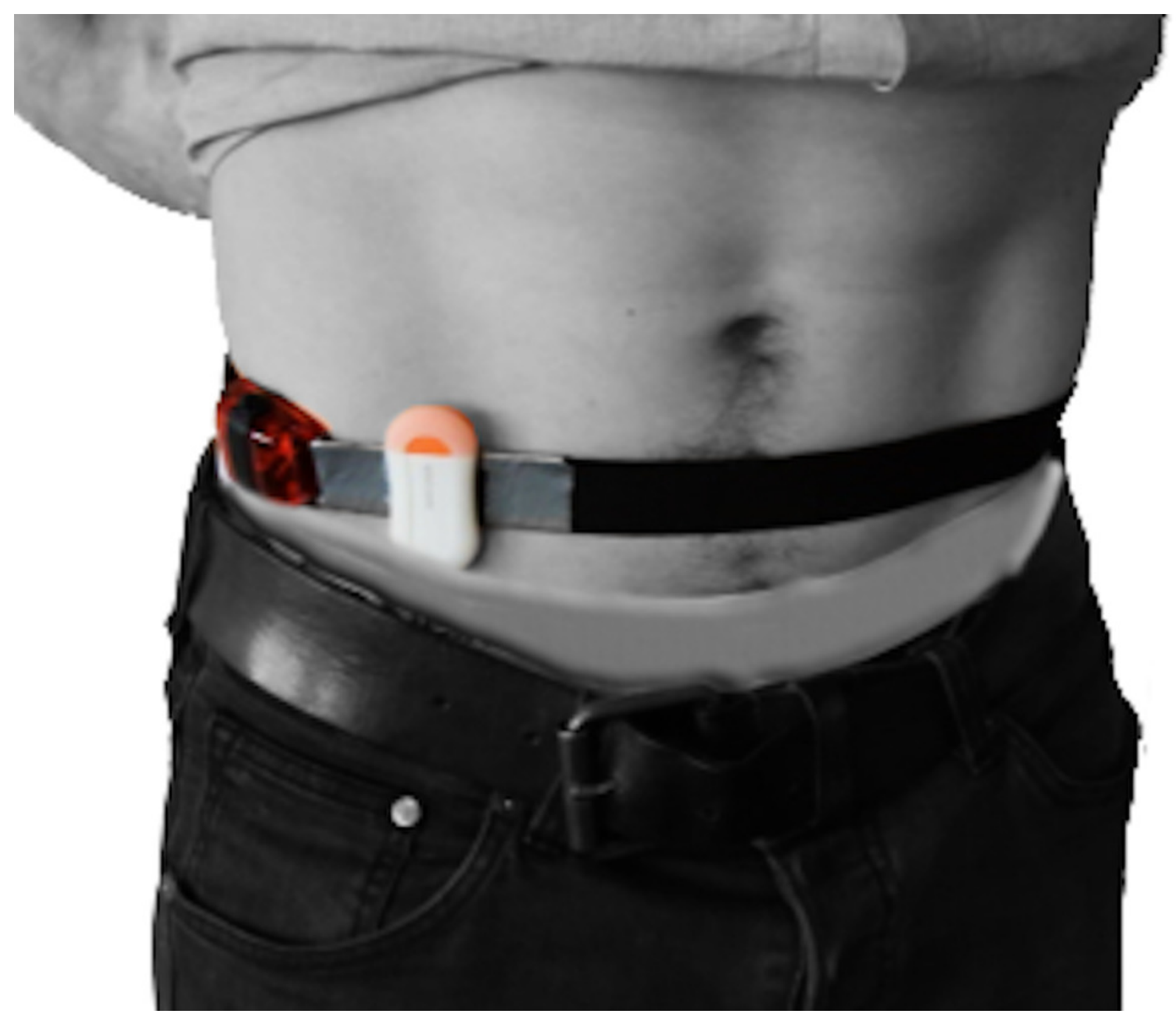


Figure 2

Bland-Altman style plot of differences between the actual number of steps and steps measured by Mother

The solid line depicts the median of differences and the dotted lines depict the $2.5^{\text {th }}$ and $97.5^{\text {th }}$ percentiles of each walking speed. The colour of the data point refers to the walking speed.

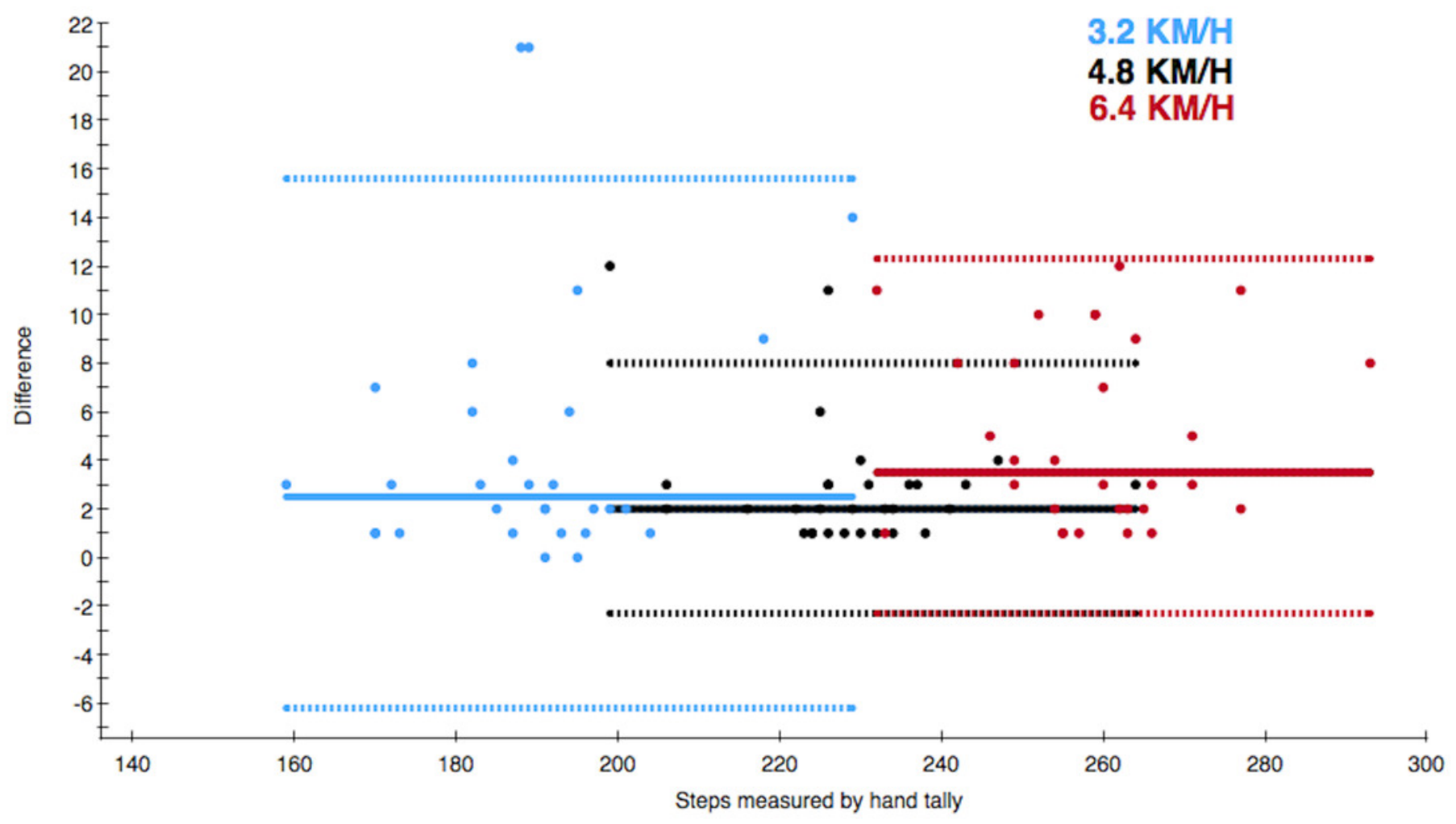


Figure 3

Bland-Altman style plot of differences between the actual number of steps and steps measured by ActiGraph

The solid line depicts the median of differences and the dotted lines depict the $2.5^{\text {th }}$ and $97.5^{\text {th }}$ percentiles of each walking speed. The colour of the data point refers to the walking speed.

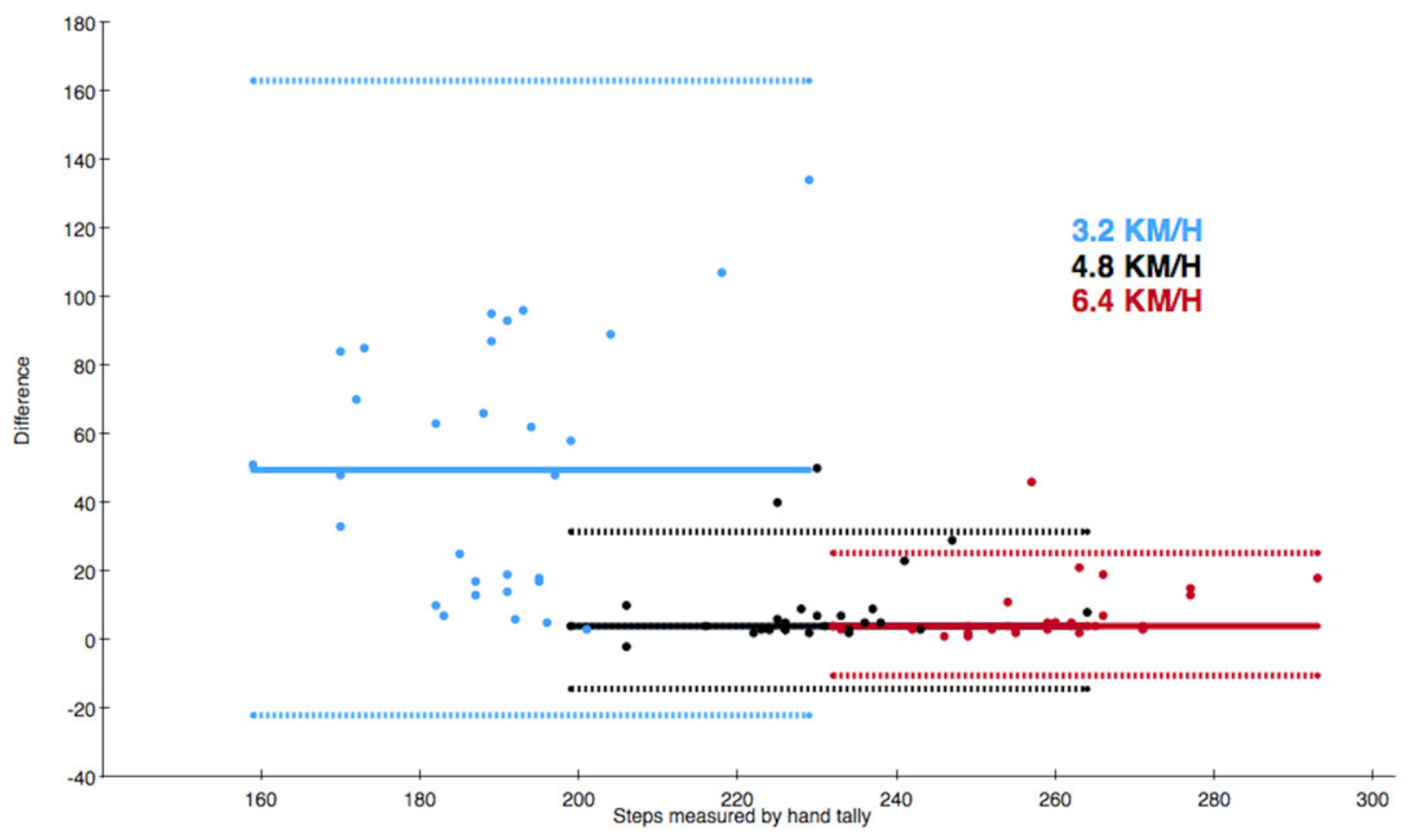

